\title{
LE RAPPORT DES BIBLIOTHÉCAIRES DE LECTURE PUBLIQUE AUX AUTEURS
}

\author{
Cécile Rabot
}

Presses Universitaires de France | Sociologie

2012/4 - Vol. 3

pages 359 à 376

ISSN 2108-8845

Article disponible en ligne à l'adresse:

http://www.cairn.info/revue-sociologie-2012-4-page-359.htm

Pour citer cet article :

Rabot Cécile, «Le rapport des bibliothécaires de lecture publique aux auteurs »,

Sociologie, 2012/4 Vol. 3, p. 359-376. DOI : 10.3917/socio.034.0359

Distribution électronique Cairn.info pour Presses Universitaires de France.

(C) Presses Universitaires de France. Tous droits réservés pour tous pays.

La reproduction ou représentation de cet article, notamment par photocopie, n'est autorisée que dans les limites des conditions générales d'utilisation du site ou, le cas échéant, des conditions générales de la licence souscrite par votre établissement. Toute autre reproduction ou représentation, en tout ou partie, sous quelque forme et de quelque manière que ce soit, est interdite sauf accord préalable et écrit de l'éditeur, en dehors des cas prévus par la législation en vigueur en France. II est précisé que son stockage dans une base de données est également interdit. 


\title{
Le rapport des bibliothécaires de lecture publique aux auteurs
}

\author{
The relation of public librarians to authors
}

par Cécile Rabot*

\section{R É S U M É}

Cet article vise à analyser la manière dont la figure de l'auteur est mobilisée et travaillée par les bibliothécaires de lecture publique au service de la définition de leur identité professionnelle. Les différents types de rapports qu'ils entretiennent avec les auteurs correspondent aux différentes manières dont ils envisagent leur métier, entre transmission de valeurs déjà constituées, participation critique à la construction de ces valeurs et animation culturelle conçue comme construction d'un espace de découverte et d'échange. L'article prend appui sur une enquête menée dans les bibliothèques de la Ville de Paris de 2005 à 2010. D'abord, dans une forme d'allégeance aux valeurs du champ, les bibliothécaires concourent à placer l'auteur dans une position sacrée et à produire la croyance dans la valeur de noms propres (I). Par ailleurs, ils entendent se poser en gardiens du temple, lectores garants de l'auctoritas et contribuant au passage du texte singulier à l'œuvre (II). Enfin dans une nouvelle posture plus ou moins assumée d'animateurs culturels, ils font venir l'auteur luimême dans le monde des livres qu'est la bibliothèque mais, ce faisant, maintiennent une division des rôles entre créateurs et récepteurs (III).

\begin{abstract}
A B STRACT
This paper aims to analyze what public librarians do with authorship and authors in order to build their professional identity. Indeed, the different kinds of relationships between librarians and authors can be connected to the different ways librarians consider their work: they have to pass on famous authors, but they also intend to contribute to build reputations and to organize cultural activities as a space of discoveries and discussions. The analysis is based on a survey conducted in the public libraries of Paris from 2005 to 2010. Firstly, librarians contribute to build the sacred position of authorship and to produce faith in the value of names (I). Moreover, they intend to be recognized as gatekeepers and to control authorship through their critical labour (II). Finally, they try to play a new role of cultural animation, organizing meetings and conferences with authors, but, doing so, they maintain the traditional division between authors and lectors (III).
\end{abstract}

MOTS-CLÉS : auteur ; écrivain ; bibliothèque ; bibliothécaire ; lecture publique; sociologie de la littérature; identité professionnelle; champ littéraire
KEYWORDS: author; authorship; public library; librarians; sociology of literature; professional identity; literary field

\footnotetext{
* Sociologue, maître de conférences à l'Université Paris Ouest Nanterre La Défense, chercheure associée au Centre Européen de Sociologie et de Science Politique (UMR 8209 Paris Panthéon Sorbonne/ CNRS/ EHESS) CESSP - 59 rue Pouchet - 75017 Paris cecile.rabot@orange.fr
} 
$\mathbf{L}^{2}$ es bibliothécaires constituent une profession très structurée et reconnue par l'État, avec des concours qui contrôlent l'accès à la profession, des progressions de carrière définies à l'intérieur de corps et de grades et une organisation professionnelle, l'Association des Bibliothécaires Français, qui, depuis sa création en 1906, a largement participé à cette reconnaissance de la profession. Mais l'identité sur laquelle s'est construit le groupe professionnel (Seibel, 1992) demeure aujourd'hui encore un objet d'interrogations, dont on trouve la trace dans la littérature professionnelle, notamment sous la plume d'Anne-Marie Bertrand (Bertrand, 2004), conservateur général et directrice de l'École Nationale Supérieure des Sciences de I'Information et des Bibliothèques. Inscrites dans une plus large « crise des identités » (Dubar, 2000), ces interrogations se nourrissent d'un triple constat : celui d'une diversité du groupe professionnel liée à la multiplicité des statuts et surtout des lieux d'exercice (avec notamment l'importante division entre bibliothèques de lecture publique $^{1}$ et bibliothèques d'étude), celui d'une méconnaissance de la profession de la part du grand public mais aussi des autres professionnels du livre, et enfin celui de mutations technologiques qui invitent à reconsidérer l'offre des bibliothèques et surtout les services proposés.

Intermédiaires dans une chaîne du livre dont les deux extrémités sont constituées par les auteurs et les lecteurs, les bibliothécaires construisent et renégocient leur identité professionnelle à travers leur rapport aux publics mais aussi à travers leur rapport aux auteurs. C'est ce second aspect que cet article se propose d'analyser. Comment les bibliothécaires construisent-ils, à travers les rapports qu'ils entretiennent avec les auteurs, une identité professionnelle à multiples facettes? Si leur mission traditionnelle fait d'eux des relais chargés de rendre accessibles les œuvres d'auteurs déjà reconnus, ne participent-ils pas aussi, via leur travail critique de sélection, à une forme de contrôle du droit d'entrée dans le champ littéraire ? (Bourdieu, 1991). Comment l'animation culturelle, aujourd'hui mise en avant dans les bibliothèques de lecture publique, participe -t-elle à reconfigurer le rapport à l'auteur en même temps que l'identité des bibliothécaires?

L'article se fonde sur une enquête menée de 2005 à 2010 dans le réseau des bibliothèques de la Ville de Paris (voir encadré) au double niveau de quelques établissements et surtout du réseau et des services centraux (Rabot, 2011b). L'enquête s'est faite par observation de réunions (séances de travail du comité « romans $»^{2}$, réunions d'équipe dans une bibliothèque, réunions de réseau entre chefs d'établissements et secrétaires des comités). Ces observations ont été complétées par une vingtaine d'entretiens approfondis (conservateurs et bibliothécaires responsables d'établissements, bibliothécaire coordinatrice du travail des comités, secrétaire du comité "romans », membres du comité « science-fiction », bibliothécaire spécialisée en littérature policière, bibliothécaires participant à l'opération «Premiers romans », bibliothécaires de la section adulte d'une bibliothèque moyenne). Enfin, des analyses quantitatives et textuelles ont été menées sur des sélections bibliographiques (présentoir "Coups de cœur », listes de « Nouveautés », fascicule «Premiers romans »).

\section{LE RÉSEAU PARISIEN DE LECTURE PUBLIQUE}

Le réseau des bibliothèques de la Ville de Paris est constitué de 58 bibliothèques de prêt de taille variable (de 105 à $4200 \mathrm{~m}^{2}$ ) autonomes dans leurs acquisitions et leurs initiatives de mise en valeur - autonomie des établissements qui est une spécificité du réseau parisien. II existe cependant des services communs centraux, notamment un service technique et des comités d'analyse de la production éditoriale répartie en domaines de spécialité. Les « mises en valeur des collections » y prennent essentiellement trois formes : celle, traditionnelle, de la bibliographie (listes ou fascicules imprimés ou en ligne), celle, largement répandue, de la mise en espace (tables ou présentoirs), celle, plus ponctuelle mais très valorisée, de l'animation. La plupart des sections adultes organisent régulièrement des animations, constituent des bibliographies thématiques et présentent

1. Pour l'essentiel prises en charge par les municipalités ou les départements et ouvertes à tous, les bibliothèques de lecture publique proposent une offre de fictions et de documentaires dans une double perspective d'accès au savoir (CSB, 1991) et au loisir. Elles constituent l'institution culturelle la plus fréquentée.
2. Constitués de bibliothécaires volontaires, en poste dans des bibliothèques du réseau, les comités d'analyse sont des groupes qui analysent la production éditoriale et présentent ces analyses dans des réunions de réseau où est élaborée une liste de propositions d'achats ensuite envoyée à chaque bibliothèque du réseau. 
trois types de sélection, sur des présentoirs dédiés : une sélection thématique, un ensemble de nouvelles acquisitions et un lot de "Nouveautés " (ouvrages achetés au niveau du réseau selon une procédure accélérée). Certaines proposent aussi une sélection de "Coups de cœur ». Par ailleurs, trois dispositifs spécifiques de mise en valeur existent au niveau du réseau : l'opération "Premiers romans " réunit quelques bibliothèques autour de la lecture exhaustive de la production de premiers romans dans le but d'en proposer une sélection critique ; les "Coups de cœur » annuels de chaque comité d'analyse, présentés sous forme de fascicules diffusés dans les bibliothèques, rassemblent les ouvrages repérés tout au long de l'année par les comités; enfin la Bibliothèque des Littératures Policières ${ }^{3}$ publie annuellement sous forme de livre une vaste sélection critique de littérature policière intitulée Les Crimes de l'annéet et fondée sur un examen exhaustif de la production du genre.

\section{Transmission}

Comme instrument fondamental d'une politique de « démocratisation culturelle » entendue d'abord comme mise à disposition des « grands auteurs » (Dubois, 1999), les bibliothécaires ont d'abord à garantir la préservation de fonds de référence dont la valeur n'est plus à prouver, et à assurer leur transmission. Mais leur rôle va plus loin : il consiste à renforcer et à prolonger ce capital symbolique en participant à la production de la croyance dans une valeur des œuvres et des auteurs censée faire l'unanimité et se passer de tout discours.

\section{L'hégémonie du nom d'auteur}

Cette perspective de transmission et de perpétuation de noms d'auteurs fonctionnant comme des valeurs reconnues conduit à un mode d'approche des textes qui rapporte chaque titre à un nom d'auteur. Le secrétaire du comité d'analyse des romans dans les bibliothèques de la Ville de Paris emploie ainsi l'expression « lire des auteurs » ("J'aime beaucoup lire des auteurs espagnols ou sud-américains hispaniques ou allemands. ») et explique que les variations dans sa pratique de lecture critique dépendent « du coup de foudre qu'[il a] ou non pour l'auteur ». Les auteurs repérés servent alors de référence pour évaluer l'originalité des auteurs inconnus ("Je compare l'auteur à un autre, surtout pour les étrangers. Je dis : "Ah oui, celui-là, par rapport à tel autre auteur mexicain, il apporte ça..." »). Le nom propre des auteurs reconnus ne réfère pas à la personne qui le porte, ou seulement de manière secondaire, mais évoque un ensemble de titres, une période historique (souvent élargie à un siècle dans une perception un peu vague résultant de la division traditionnelle en siècles de l'histoire littéraire et de ses manuels), un genre (un auteur classique est souvent associé à un genre donné, fût-ce au prix d'une oblitération de ses autres productions) et surtout un niveau de valeur et une reconnaissance partagée de ce niveau de valeur.

La logique du capital symbolique de l'auteur ne joue pas seulement pour les auteurs canoniques mais de manière plus générale pour les auteurs déjà repérés et légitimés par les bibliothécaires et par d'autres instances. Le nom propre de l'auteur suffit alors à provoquer la décision d'acquisition de tel ou tel ouvrage, d'autant plus que la décision ne peut se fonder sur l'examen critique du livre considéré. Il joue un rôle important lors des réunions d'équipe ${ }^{5}$, organisées dans la plupart des établissements dans le but d'une réflexion collective sur les projets d'acquisition de la bibliothèque. L'examen des nouveautés éditoriales s'y fonde en effet la plupart du temps sur les derniers numéros de l'hebdomadaire Livres Hebdo, qui recense l'ensemble des dernières publications des éditeurs français, mais en ne fournissant que les informations bibliographiques élémentaires assorties d'une phrase de présentation. Ces éléments suffisent parfois à cerner un documentaire mais s'avèrent particulièrement insuffisants pour se faire une idée d'un texte littéraire. Les auteurs déjà connus émergent alors nettement et s'imposent avec une sorte d'évidence aux bibliothécaires, qui font précéder leur nom d'un article défini indiquant une réputation déjà faite : le Paul Auster est naturellement sélectionné, comme le Paul Celan et le Paul Veyne, auteurs qu'on ne présente plus, tandis que les auteurs inconnus des bibliothécaires
3. Bibliothèque spécialisée du réseau parisien, installée dans le $5^{\mathrm{e}}$ arrondissement, la Bilipo reçoit un exemplaire du dépôt légal de tout ce qui se publie en matière de littérature policière et met ce fonds, ainsi que des documents d'archives, à disposition du public pour une consultation sur place.
4. Éditions Paris bibliothèques.

5. Les lignes qui suivent s'appuient sur l'observation d'une réunion d'équipe organisée dans une grande bibliothèque du réseau parisien le 8 mars 2007. 
restent dans l'ombre, noyés dans la masse des productions littéraires impossibles à juger en aveugle.

Cette logique " auctoriale » se retrouve dans les classements par nom d'auteurs mais aussi dans les choix éditoriaux des bibliothécaires. Elle conduit d'abord à écarter les versions tronquées ou adaptées qui ont pourtant eu leur heure de gloire dans l'optique de faire découvrir les " grands auteurs » aux publics scolaires ou populaires (Olivero, 1999) : c'est le texte original et intégral, tel qu'il a été conçu et écrit par l'auteur, qu'il s'agit de mettre à disposition. Bien plus, il s'agit de proposer, quand elle existe, une édition en "œuvres complètes » qui réunit l'ensemble des textes d'un auteur (du moins des textes reconnus $^{6}$ ) sous un nom qui les fédère, et qui est supposée favoriser la circulation entre les textes. C'est ainsi que la "Bibliothèque de la Pléiade » des éditions Gallimard, qui constitue le couronnement du processus de consécration (Gleize \& Roussin, 2009), est particulièrement en faveur dans les bibliothèques parisiennes ${ }^{7}$. Les titres singuliers y sont escamotés derrière le nom d'auteur qui figure seul sur le dos du livre.

À l'inverse, le travail des autres acteurs de la chaîne du livre, notamment de l'éditeur ou du traducteur, est rarement pris en considération, malgré son rôle dans la constitution des textes et de leur valeur (Casanova, 2002). L'observation des éditions choisies par les bibliothécaires pour les auteurs classiques ne révèle aucun intérêt général pour des traductions revisitées ou des lectures renouvelées des textes. Si chaque bibliothèque est par exemple attachée à proposer les comédies de Plaute, la traduction de La Marmite par Florence Dupont ${ }^{8}$ n'est disponible que dans deux bibliothèques du réseau. Le plus souvent, l'acquisition de telle ou telle traduction semble résulter de la coïncidence entre le besoin de renouveler des livres perdus ou abîmés et l'existence d'une nouveauté éditoriale bien plus que d'un choix raisonné évaluant les partis-pris de traduction. Ce qui importe, c'est d'avoir ces auteurs classiques au catalogue, sous une forme ou une autre.

\section{Une posture d'allégeance}

Une telle importance donnée au nom d'auteur renvoie à une approche lettrée, qui résulte d'une socialisation familiale et surtout scolaire et professionnelle, mais qui n'est pas universelle. Ainsi, le regroupement de plusieurs titres sous un nom d'auteur dans un volume d'« œuvres », familier au lecteur lettré ou professionnel, n'est pas évident pour tous, comme le constate une responsable d'établissement, conservatrice de 53 ans, plusieurs fois admissible à l'École des Chartes, à propos d'un contractuel récemment recruté sans expérience préalable du monde du livre :

\begin{abstract}
"Même moi j'ai été un peu désarçonnée en début de semaine quand il est arrivé avec un Pléiade, [Elle rit] cet objet très étrange qu'est un volume de la Pléiade ! Et il m'a posé une question. Après je me suis dit : mais c'est vrai, moi j'ai tellement vécu là-dedans... II me dit: "Mais dans ces livres-là, il y a plusieurs titres d'un même auteur, n'est-ce pas?" [Elle rit] Eh bien oui ! Je ne sais plus ce qu'il cherchait, c'était dans les œuvres romanesques de Giono, donc il avait repéré qu'il y avait plusieurs volumes de Giono dans le volume 1 , mais qu'il pouvait y avoir plusieurs tomes qui dans une autre... Enfin, il n'est pas bête du tout, en plus, hein ! Je veux dire, il se pose les bonnes questions, mais alors quelquefois ça fait un peu étrange quoi ! » (entretien du 29 juillet 2010)
\end{abstract}

En particulier, l'approche par nom d'auteur n'a rien d'automatique pour les lecteurs des classes populaires et pour les jeunes lecteurs, qui se focalisent sur le contenu des livres et raisonnent plutôt par titres et par collections sans mémoriser les noms d'auteurs (Thiesse, 1984, p. 35 ; Bahloul, 1988) divergence d'approche qui n'est peut-être pas étrangère au sentiment de malaise que certains éprouvent en bibliothèque devant un ordre qui les déroute.

À l'inverse, la logique auctoriale, amenant à rapporter chaque livre singulier au nom de son auteur, est devenue la norme de l'approche lettrée de la littérature, comme l'a souligné Michel Foucault :

«Les discours «littéraires» ne peuvent plus être reçus que dotés de la fonction auteur : à tout texte de poésie ou de fiction on demandera
6. "On peut dire que l'auteur classique par excellence est l'auteur intégralement intégré. Intégral parce qu'il a droit à l'édition en CEuvres complètes, que le moindre de ses brouillons devient même objet de quête et de publication (effet maximal de perpétuation). Et intégré parce que sur l'ensemble de l'œuvre les aspects mis en avant et ceux qui se trouvent occultés sont nettement distincts. C'est Molière interprété en négligeant les comédiesballets et les farces, Racine ramené à ses seules tragédies, et parmi elles à quelques-unes, et de nos jours à Phèdre surtout ; c'est Vallès ramené à L'Enfant. » (Viala, 1993, p. 26)

7. Le réseau parisien comporte près de 15000 volumes de cette collection.

8. Plaute (2001), La Marmite ; suivi de Pseudolus / trad. du latin et préf. par Florence Dupont, Arles, Actes Sud, coédité avec Leméac (Montréal). 
d'où il vient, qui l'a écrit, à quelle date, en quelles circonstances ou à partir de quel projet. Le sens qu'on lui accorde, le statut ou la valeur qu'on lui reconnaît dépendent de la manière dont on répond à ces questions. Et si, par la suite d'un accident ou d'une volonté explicite de l'auteur, il nous parvient dans l'anonymat, le jeu est aussitôt de retrouver l'auteur. L'anonymat littéraire ne nous est pas supportable ; nous ne l'acceptons qu'à titre d'énigme. La fonction auteur joue à plein de nos jours pour les œuvres littéraires. » (Foucault, 1969, p. 828)

En mobilisant une telle approche, les bibliothécaires adoptent un schème de perception « lettré » transmis par l'enseignement secondaire et universitaire. Ils manifestent ainsi la manière dont ils ont été socialisés à la littérature par leur formation (la plupart ont suivi des études de lettres et sciences humaines). Mais surtout, en s'appropriant les schèmes de perception et les valeurs qui y dominent, ils font acte d'allégeance au champ littéraire, de manière à prouver qu'ils y appartiennent, ce que leur contestent la plupart des autres acteurs du champ. Parler par noms d'auteurs, c'est montrer qu'on sait décrypter les signes qu'ils constituent, qu'on partage suffisamment les repères communs pour pouvoir " parler des livres que l'on n'a pas lus » (Bayard, 2007), qu'on reconnaît les classements et donc qu'on fait partie d'une communauté partageant des valeurs.

Cette approche n'est pas partagée par tous les bibliothécaires, en particulier par ceux qui manifestent une distance plus grande avec l'univers académique et le modèle de lecture formaliste ${ }^{9}$ qui lui est attaché. Ce rejet de la posture lettrée prend un sens institutionnel quand on sait que la lecture publique s'est construite en grande partie contre un certain modèle scolaire (Chartier \& Hébrard, 2000 ; Tarin, 2006). Mais il est frappant que cette mise à distance de la posture lettrée est souvent le fait de bibliothécaires qui ne se sentent guère à l'aise avec elle personnellement et dont les trajectoires scolaires ont été marquées par des déceptions (dégoût du français au lycée, khâgne mal vécue, échec aux concours de l'enseignement).

L'approche lettrée est cependant valorisée dans la profession et dans le réseau des bibliothèques de la Ville de Paris. En témoigne par exemple la place occupée dans le réseau par le secrétaire du comité d'analyse des romans. Malgré son grade de bibliothécaire adjoint spécialisé (inférieur donc à celui de bibliothécaire, lui-même inférieur à celui de conservateur), il participe aux décisions d'acquisitions dans la bibliothèque moyenne où il travaille. Surtout, il bénéficie d'un capital symbolique au sein du réseau par le rôle de secrétaire du comité d'analyse des romans qu'il exerce depuis de nombreuses années et qui lui permet de présenter les romans nouvellement publiés à l'ensemble des responsables d'établissements (qui sont pour la plupart des conservateurs). La posture par laquelle il légitime sa position est une posture «lettrée » : il écrit lui-même des nouvelles (publiées par de petits éditeurs), fréquente des clubs de poésie et, au sein du comité d'analyse des romans des bibliothèques de la Ville de Paris, incarne l'approche la plus «littéraire » avec des critiques qui mettent en avant les propriétés stylistiques des textes quand d'autres membres du comité privilégient l'intrigue. Ainsi, même si elle n'est pas le fait de tous les personnels, la tendance à lire des auteurs plutôt que des livres singuliers semble une norme professionnelle implicite.

\section{Un idéal de consensus}

La posture lettrée implique aussi la reconnaissance des valeurs partagées dans le champ littéraire. Elle suppose que ces valeurs reposent sur le talent des auteurs et les propriétés des œuvres, qu'il s'agit de les reconnaître et que les différentes instances doivent finir par trouver un consensus sur la définition d'un ensemble de "grands auteurs ». C'est ainsi que pour les bibliothécaires s'imposent un certain nombre d'auteurs considérés comme " de référence » ou « incontournables », dont l'absence dans les collections serait malvenue. Les auteurs du canon, dont l'institution scolaire assure la transmission ou au moins une connaissance élémentaire permettant d'en parler sans forcément les avoir lus (Bayard, 2007), assortie de la reconnaissance de leur valeur, s'imposent ainsi dans les bibliothèques de lecture publique avec l'évidence que leur confèrent leur niveau élevé de légitimité et leur statut symbolique d'auteurs en théorie connus et reconnus de tous. II ne s'agit pas pour les bibliothécaires de tenter d'élargir ce canon à des auteurs du passé moins connus : une étude de la présence dans les bibliothèques parisiennes des auteurs mis au programme de l'agrégation de lettres révèle que seuls y sont 
présents les auteurs les plus connus, ceux que l'on retrouve couramment dans les manuels scolaires. La consécration passe par le consensus : le statut de " grand auteur » requiert une reconnaissance par un nombre maximal d'instances.

Les bibliothécaires ne se contentent pas de participer à la reconnaissance générale de la valeur des classiques, ils visent un semblable consensus pour des auteurs contemporains qui n'ont pas encore atteint ce statut. II ne s'agit pas pour eux de proposer des sélections distinctives mais de participer à une consécration collective aux côtés des critiques de presse, des jurys de prix littéraires et des libraires. La responsable de la sélection des « Nouveautés » dans le réseau des bibliothèques de la Ville de Paris, une bibliothécaire de 42 ans, se félicite ainsi de réussir à sélectionner comme "Nouveautés » la plupart des ouvrages récompensés ensuite par un prix littéraire ( Les prix littéraires, on est très fort ! »). De même, le secrétaire du comité d'analyse des romans considère comme un défaut de n'avoir pas porté attention à un auteur qui reçoit par la suite un grand prix ou un accueil très favorable du public, même s'il met aussi à distance ces formes de reconnaissance :

\footnotetext{
« Une année, par exemple, on avait éliminé en réunion le Renaudot, enfin le livre qui a eu le Renaudot après. On l'avait vu la semaine d'avant, il a eu le Renaudot après et on l'avait éliminé. Enfin, ça ne veut rien dire parce que les prix, on sait bien qu'il y aussi un côté parisianiste... Mais enfin on était un peu vexé, quand même ! [I/ rit] Par contre, L'Élégance du hérisson ${ }^{10}$, on l'avait remarqué... on l'avait déjà bien remarqué... » (entretien du 15 avril 2008)
}

La responsable de la sélection des imprimés ${ }^{11}$ pointe aussi la coïncidence visée entre les listes de propositions d'achats, dont elle supervise l'élaboration, et les livres mis en avant par les libraires : elle évoque ainsi sa satisfaction à constater que trente romans désignés comme "Coups de cœur des libraires» avaient très largement été retenus par les bibliothécaires, même si le recul que lui donne l'expérience (elle est en fin de carrière) l'amène aussi à interroger ce désir de consensus :

« On était bon à 95 \%, c'est-à-dire que, pour beaucoup, on avait passé ces coups de cœur de libraires en Nouveautés et, pour les autres, on les avait sélectionnés sur listes [de propositions d'acquisitions]. Donc c'est vrai, quelque part ça fait plaisir, parce qu'on se dit qu'on n'est pas complètement à côté de la plaque. On est, comment dirais-je, on est sur les rails : on est comme tout le monde. C'est peut-être aussi un défaut, vous pourriez dire, mais voilà, c'est le cœur du problème peut-être : est-ce qu'on est trop consensuel ou... Voilà. » (entretien du 4 juillet 2007)

Le dispositif des « Nouveautés » consiste ainsi à commander, avant parution et sur la base d'une avant-critique, des ouvrages d'auteurs occupant des positions dominantes de la République mondiale des lettres (Casanova, 1999) (en termes de pays d'origine, de langue d'origine, de genre et de maison d'édition $^{12}$ ), repérés par la critique et par les bibliothécaires et ayant connu un certain succès auprès du public. Le capital symbolique accumulé autour du nom d'auteur fonctionne comme une garantie de valeur et une assurance de succès : il suscite l'attention des critiques de presse, favorise donc la publication de critiques, qui donnent une visibilité au livre et font naître l'intérêt des lecteurs.

De même, les «Coups de cœur » redoublent ou relaient souvent une consécration conférée par d'autres instances de légitimation. La plupart d'entre eux a reçu une critique de presse favorable. Beaucoup sont des livres d'auteurs qui ont été sélectionnés pour un ou plusieurs prix littéraires, voire qui les ont remportés ${ }^{13}$. La mise en avant sur un présentoir de bibliothèque apporte à un livre déjà légitimé un surcroît de crédit, tout en assurant l'effet d'écho qui semble nécessaire à la décision de lecture. L'intérêt pour l'auteur se construit dans l'accumulation des recommandations ou des évocations. Le livre est alors censé parler de lui-même, d'où l'absence de notice critique accompagnant les sélections.

\section{Inscrire dans la durée : constituer un fonds d'auteurs}

Relayer, c'est aussi inscrire les auteurs dans une durée qui est celle de leur trajectoire mais aussi celle, plus longue, de l'histoire littéraire. Devant l'accélération de l'édition et de la rotation
10. Muriel Barbery (2006), L'Élégance du hérisson, Paris, Gallimard. Longtemps dans les meilleures ventes.

11. Ce poste, dans un des services centraux du réseau, inclut les relations avec les libraires fournisseurs, la coordination des comités d'analyse et l'organisation des procédures de sélection au niveau du réseau (de la définition de "l'office » envoyé par les libraires à l'envoi de listes de propositions d'acquisitions aux bibliothèques du réseau).
12. Sur les six mois de "Nouveautés " étudiés, $90 \%$ des titres sont des romans, $62 \%$ des livres traduits de l'américain, $70 \%$ des publications de grands ou très grands éditeurs (publiant plus de 80 titres par an).

13. Un quart des auteurs de la sélection de « Coups de cœur » analysée ont été lauréats d'un prix. 
des livres en librairie, la bibliothèque s'affirme comme un lieu de constitution d'une mémoire en continuant à proposer et à mettre en valeur non seulement les classiques mais aussi les ouvrages repérés pour leur intérêt par la critique ou le public, mais depuis longtemps remplacés par d'autres sur les tables des libraires. Les bibliothécaires se démarquent ainsi d'une critique de presse tout entière focalisée sur les dernières publications, sans cesse à l'affût de renouvellement et prise dans une pression d'actualité particulièrement visible au moment de la rentrée littéraire (les quelque sept cents romans publiés à chacune des dernières rentrées littéraires constituent une gageure qui exclut la possibilité d'y adjoindre d'autres lectures « de rattrapage " d'ouvrages plus anciens). À côté de productions récentes, ils tiennent à mettre en avant des valeurs sûres établies au fil des dernières années mais toujours valables : loin de se cantonner à la production des deux ou trois derniers mois, la vie littéraire s'inscrit dans la durée.
Par sa temporalité propre, intermédiaire entre la temporalité courte de l'édition et le temps long du canon, la bibliothèque constitue un relais important participant à inscrire les auteurs dans une durée susceptible de précéder leur éventuelle classicisation. Les ouvrages mis en valeur dans certaines sélections des bibliothèques, notamment les "Coups de cœur", relèvent massivement d'une temporalité médiane qui n'est plus celle de l'actualité éditoriale sans être encore le temps long du canon (voir diagramme ci-dessous).

En continuant à mettre ces livres en avant une fois retombé l'effet médiatique engendré par leur consécration, les bibliothécaires leur évitent de tomber dans l'oubli : non contents de conserver, conformément à leur mission première, ils participent à préserver la valeur d'usage des ouvrages sortis de l'actualité. Le dispositif des «Coups de cœur » vise par ailleurs à faire découvrir d'autres titres moins connus, antérieurs ou

\section{Répartition d'une sélection de "Coups de cœur » observée dans une bibliothèque parisienne moyenne en fonction de leur date de publication ${ }^{14}$}

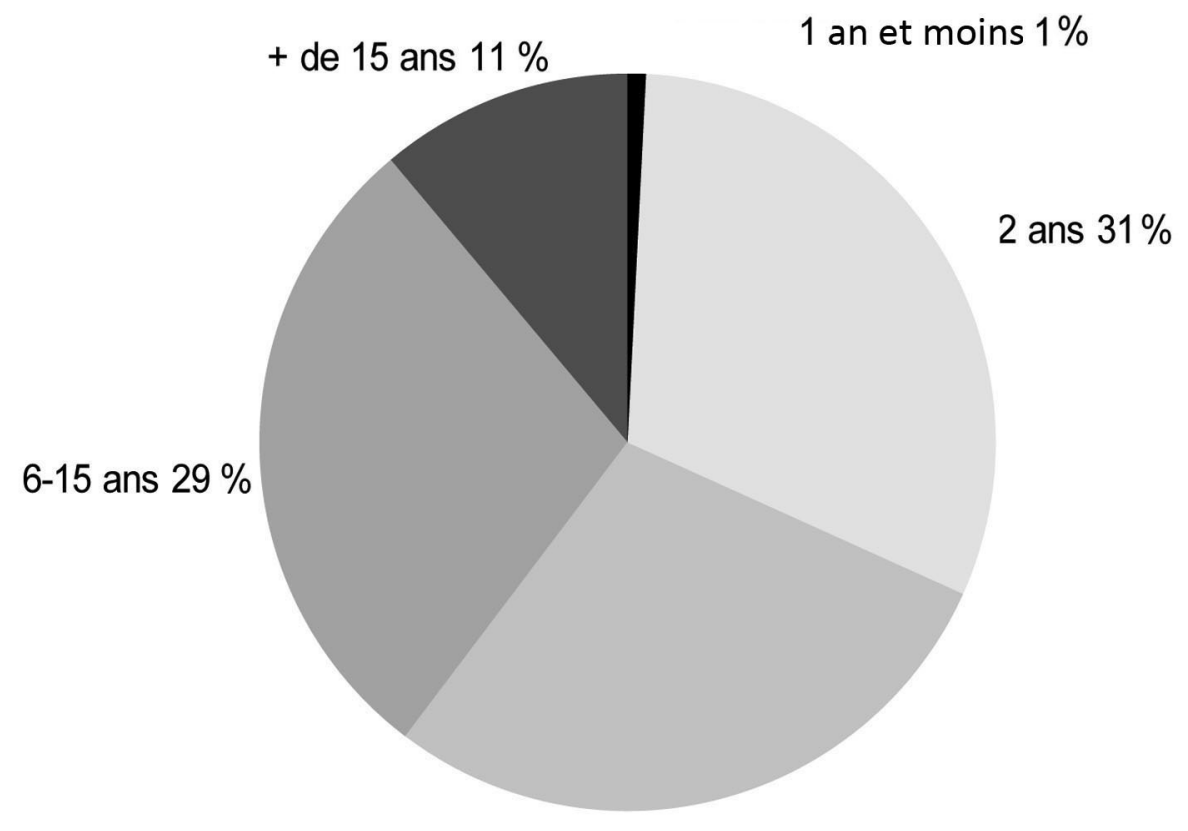

$3-5$ ans $29 \%$

14. Le corpus considéré, constitué par une série de relevés systématiques 
postérieurs, de ces auteurs (Rabot, 2011a). Par les premiers, les bibliothécaires montrent qu'ils n'ont pas attendu la consécration générale pour découvrir cet auteur. Par les seconds, ils prouvent qu'ils continuent à suivre les auteurs repérés et refusent l'éphémère du succès.

Inscrits dans une logique de mise en valeur du fonds, les présentoirs "Coups de cœur», comme les tables thématiques, présentent ainsi des œuvres en voie de patrimonialisation sans toutefois faire encore partie du canon académique stricto sensu. On y trouve par exemple quelques auteurs français de l'entre-deux-guerres ou d'après-guerre et surtout des classiques étrangers, américains, européens ou japonais, ou encore des œuvres devenues les classiques des genres en voie de légitimation (comme le roman policier). En constituant ainsi un fonds de "valeurs sûres » et de «bons auteurs », qui ont reçu la légitimation de différentes instances, la bibliothèque de lecture publique est susceptible de jouer un rôle de tremplin pour l'accès des ouvrages sélectionnés au statut de classiques, ce qui fait d'elle une instance importante du champ littéraire, quoique souvent ignorée d'éditeurs centrés sur l'actualité des publications, voire sur le futur de leur plan de publications.

\section{Jugement critique}

Au-delà de cette fonction de transmission qui concerne les auteurs à la réputation déjà faite, les bibliothécaires revendiquent, dans une perspective de division du travail d'expertise avec la profession d'enseignant (Abbott, 1988), une identité fondée sur une expertise critique : ils entendent non seulement confirmer une auctorialité construite ailleurs mais participer à la construire. Ce travail d'auctorialisation a par conséquent à se déployer plus spécialement dans les domaines où la légitimité des productions n'est pas complètement acquise, que cela tienne au statut de l'auteur, encore peu (re)connu, ou à celui du genre, en voie de légitimation (Parmentier, 1986). C'est donc significativement le premier roman, le roman policier, la science-fiction / fantasy et la bande dessinée qui font l'objet d'un travail d'analyse et de mise en valeur tout particulier de la part des bibliothécaires de lecture publique, qui trouvent là un moyen de participer aux luttes pour la définition de la valeur littéraire.

\section{Repérer les auteurs à naître ou le pari sur l'œuvre à venir}

L'opération "Premiers romans » des bibliothèques de la Ville de Paris s'inscrit dans cette perspective. Elle consiste en une analyse de l'ensemble des premiers romans de langue française publiés par des éditeurs français dans l'année écoulée ${ }^{15}$ et en l'établissement d'une sélection critique retenant environ un premier roman sur six. Cet examen exhaustif (chaque livre est lu intégralement par plusieurs lecteurs) s'impose d'autant plus que le premier roman surgit dans le monde éditorial dépourvu de «nom d'auteur » constitué : le nom propre inscrit sur la couverture ne renvoie encore à aucune réalité dans le champ littéraire et ne peut donc fonctionner comme une garantie de valeur. Si le paratexte éditorial peut guider le jugement, le regard qu'on porte au premier roman est en revanche vierge de toute représentation préalable de l'auteur, comme le souligne une bibliothécaire engagée depuis plusieurs années dans l'opération «Premiers romans » :

\footnotetext{
« [Dans le cas d'un premier roman] il n'y a pas de suite pour l'instant, on ne connaît rien des œuvres antérieures de l'auteur : on arrive complètement... j'allais dire vierge mentalement. Enfin, on n'a pas d'a priori psychologique, ni de stéréotype et on prend le livre tel qu'il est. [...] c'est comme un enfant qui ne saurait pas lire, il ne va pas dire: "Ah, je voudrais un auteur..." Enfin, sauf [...] quand il a déjà pas mal lu, mais au départ, il prend le livre parce que quelque chose l'attire dans le livre. » (entretien du 8 avril 2008)
}

La lecture du premier roman semble à cet égard se passer plus volontiers de mise en contexte, donc requérir moins de capital culturel, ce qui est peut-être un des intérêts que peut trouver cette bibliothécaire à s'investir dans le premier roman alors qu'elle a un relatif sentiment d'illégitimité culturelle : d'abord bibliothécaire pour la jeunesse (donc spécialisée dans un type de livres moins légitimes que la littérature générale adulte), arrivée en fin de carrière dans une grande bibliothèque disposant d'un important fonds de niveau universitaire, cette bibliothécaire manifeste une certaine timidité devant la " grande littérature », qui lui échappe en partie, et trouve dans le premier
15. Soit un total de deux cents livres environ. Des accords avec les éditeurs, y compris petits (éditions L'Escarbille ou Bleu autour par exemple), permettent d'accéder à la production d'une manière à peu près exhaustive grâce à l'envoi des livres en "service de presse ». 
roman un moyen d'aborder la littérature adulte et de se constituer une position dans l'équipe.

Pour faire entrer un texte dans la catégorie "littérature », i.e. pour faire d'un texte une œuvre, il s'agit de le référer à un auteur. Quand l'auteur n'existe pas encore comme tel, c'est le travail critique qui permet de faire d'un écrivant un écrivain, en partant des textes eux-mêmes. L'écriture constitue alors un critère essentiel à l'évaluation des prétendants à l'entrée dans le champ, comme le suggère le secrétaire du comité "romans » qui étend ici au premier roman ce qui constitue un de ses critères dans l'analyse de la production romanesque en général : c'est le « style » ou ce « quelque chose qui se détache par rapport au lot commun » que le bibliothécaire expérimenté sait percevoir ( "On le détecte, on le détecte ») et qui permet de discriminer parmi les primo-romanciers ceux qui deviendront des auteurs qui «tiennent la route », c'est-à-dire qu'on « voit continuer après ». Le premier roman constitue à cet égard une étape décisive dans l'accès à l'auctorialité (Abensour \& Legendre, 2012), non pas tant par sa publication elle-même que par sa réception, qui constitue en quelque sorte un second droit d'entrée :

\footnotetext{
"Comme c'est un premier texte, il faut quand même qu'il fasse vraiment ses preuves auprès des lecteurs. Je pense qu'on est encore plus exigeant pour un premier roman parce qu'on se dit: il faut qu'il passe le cap des lecteurs, parce que les lecteurs nous disent souvent : il y a beaucoup de déchets, ah oui les premiers romans, ça sert de galop d'essai. » (entretien du 8 avril 2008)
}

L'opération « Premiers romans » vise surtout à repérer ceux qui deviendront des auteurs à part entière, c'est-à-dire qui publieront d'autres textes. Le premier roman est lu dans la perspective de ceux qu'il laisse présager et de la trajectoire auctoriale qu'il inaugure. La bibliothécaire précédemment citée pointe cette dimension d'avenir : "Quand on trouve que c'est valable, il faut l'encourager, il faut montrer qu'on attend encore des choses de lui. » Elle établit ainsi une distinction claire entre un titre isolé, qui n'est jamais qu'une promesse, et une œuvre, qui est la condition de l'auctorialité et de la consécration :

« II faut qu'il y ait un potentiel, un devenir. II ne faut pas que ce soit déjà tout bien fait. Parce qu'on sent que c'est encore... que ce n'est pas dans le moule, mais bon ils ont posé des jalons, et puis après on voit si ça marche ou pas. [...] On ne peut pas non plus donner le Prix Goncourt à des personnes qui sont en gestation d'auteur. Enfin, vraiment, c'est évident, n'importe qui dirait bien ça, hein, [...] ce n'est pas parce qu'on va écrire un livre, même s'il est très très bien écrit, qu'on peut être récompensé pour une œuvre. » (ibid.)
Par ce pari sur l'avenir, les bibliothécaires participant à cette opération entendent faire partie de ceux qui ont déniché tel auteur avant qu'il soit bien connu et un peu galvaudé. Ce repérage à la source constitue un fort enjeu en termes de place de la profession de bibliothécaire dans le champ littéraire, en particulier par rapport à d'autres instances critiques.

\section{L'attention à la "patte» ou la responsabilité critique}

Le suivi des auteurs n'est non plus pas inconditionnel. Les nouvelles publications d'auteurs déjà connus sont évaluées par rapport aux publications précédentes du même auteur qui constituent son « horizon d'attente» (Jauss, 1978). Pour rester à la hauteur, l'auteur ne peut pas se contenter de réutiliser les techniques éprouvées. II doit se renouveler sous peine de perdre le capital symbolique précédemment acquis :

\footnotetext{
«Moi j'ai lu quelquefois des nouveautés, bon, c'était le x ${ }^{\text {ième }}$ roman... oh la la, on sent qu'ils n'ont plus rien à dire ! Ils continuent à écrire parce que c'est bien écrit, c'est bien léché, c'est parfaitement... c'est tout trop bien, c'est tout trop bien ! [...] il y a des grands auteurs qui ne sont plus intéressants. Même il y en a qui arrivent à... je ne sais pas comment ils font pour être publiés : ils ne captivent plus du tout le public, ou alors celui qui lit ça, c'est vraiment par snobisme qu'il peut dire que c'est bien. » (ibid.)
}

Constituer un auteur, c'est en effet juger de la cohérence et de la permanence de valeur de ses différentes publications (Foucault, 2001) de manière à y faire apparaître une identité et une évolution : ce nouveau texte est-il à la hauteur des précédents ? Comment s'inscrit-il dans une trajectoire dont le sens se constitue au fur et à mesure et dans laquelle chaque texte est susceptible d'éclairer les autres? Qu'y retrouve-t-on de la « patte » de l'auteur, c'est-à-dire de ce qui fait son style et, partant, son identité ? Une bibliothécaire de la Bibliothèque des littératures policières, spécialisée dans l'analyse des nouveautés éditoriales dans ce domaine, insiste sur cette importance du style, participant ainsi à intégrer le roman policier dans la littérature, donc à légitimer un genre encore parfois suspecté de n'être qu'un produit de divertissement :

\footnotetext{
"Un livre bien écrit peut être très bien écrit, c'est-à-dire avec un style littéraire particulier, personnel: Proust n'écrit pas comme Sartre, hein. Bon, ils écrivent très bien tous les deux [Elle rit] mais ce n'est pas le même style. Donc là il y a des styles différents. Au niveau du polar, Jean-Patrick Manchette n'écrit pas comme... je ne sais pas moi, Jean-Bernard Pouy, pour prendre deux contemporains: ils écrivent très bien tous les deux, mais ce n'est pas le même style, pas
} 
la même patte si vous voulez. Et puis alors vous avez les livres mal écrits. Mal écrits, point. C'est tout, c'est mal écrit. Je ne dois pas en avoir là sous la main parce qu'en général je m'en débarrasse très vite, mais il y a vraiment des livres qui ne sont pas lisibles ! Voilà. Ce n'est pas une question de style, c'est vraiment que ce n'est pas lisible. Et quelquefois justement ils ne sont pas lisibles parce que l'auteur veut faire style, hein. Voilà. Tout le monde n'est pas Proust ou Jean-Patrick Manchette! » (entretien du 3 août 2007)

Le jugement critique, appuyé sur une compétence et sur une expérience, assure ainsi le contrôle du droit d'entrée dans le champ littéraire, faisant des bibliothécaires des gate-keepers admettant certains producteurs de textes dans le monde des auteurs et en rejetant d'autres. Est digne du titre d'auteur celui qui fait preuve d'une maîtrise en même temps que d'une singularité par laquelle il se distingue de l'horizon d'attente sur lequel il s'inscrit et qu'il participe à enrichir. L'innovation attendue concerne le style, une vision incarnée dans une écriture, c'està-dire dans le travail formel du langage. Au contraire, ceux qui écrivent des textes jugés commerciaux, réutilisant des ficelles connues au lieu d'innover, ne sont pas considérés comme de véritables auteurs, dignes d'appartenir au champ littéraire.

\section{Une posture de critique}

À travers cette attention au style et à la trajectoire des auteurs, les bibliothécaires revendiquent une capacité à lire les textes, à percevoir l'originalité d'un style ou d'une vision, à rapprocher et à comparer, c'est-à-dire à faire un travail de critique littéraire. Ce qui suppose d'abord une pratique de lecture qui ne va pas de soi dans l'activité professionnelle (à quelques exceptions près, poste spécifique ou heures concédées pour participer à un comité de lecture, la lecture est renvoyée au temps privé). Mais la lecture critique est vue comme la condition d'une autonomie de jugement, à l'égard notamment de critiques de presse souvent décriées. C'est donc une identité professionnelle (de sélectionneur libre et de critique) et une manière d'exercer le métier (juger de première main plutôt que s'en remettre à des experts) qui sont ici en jeu.

Une bibliothécaire participant à l'opération «Premiers romans » privilégie ainsi, surtout pour les auteurs inconnus que sont en général les primo-romanciers, une lecture attentive et intégrale :

"Quand je lis un premier roman, je lis tout tout tout. Je ne vais pas me dire: "Ah ben tiens, je passe deux trois pages". Ça je n'aime pas parce que c'est comme si j'avais... Enfin, pour moi, ce n'est pas honnête, quoi, vis-à-vis de la personne, surtout que bon, là il faut que j'en rende compte moi-même, [mais] même si je ne vais pas faire le résumé, l'analyse, la critique et l'appréciation générale, je veux quand même tout lire par respect de l'écrivain. Voilà. - Sinon, c'est une trahison? - Oui. [Elle rit] Donc là je lis tout le livre, [...] je ne suis pas du genre à lire dans les dix premières pages et à dire : Ah non non, ça vaut rien. Parce que moi-même je n'écris pas, donc je ne peux pas juger trop... Ah non non, ça ça ne vaut rien, déjà écrit, déjà dit, pas nouveau, clichés, machin... je vais le voir que ce n'est pas bien, mais je vais quand même le lire, surtout si c'est le premier roman. » (entretien du 8 avril 2008)

La mobilisation d'un vocabulaire éthique (honnêteté, respect) permet ici de justifier une pratique que l'on peut aussi analyser comme un manque d'assurance et une forme de « bonne volonté culturelle» (Bourdieu, 1979) : lire attentivement et in extenso est alors le signe d'une position dominée dans le groupe professionnel et d'une aspiration à une position plus élevée.

Un autre bibliothécaire évoque lui aussi la nécessité de lire les livres ou en tout cas d'en faire une analyse de première main, qu'il présente comme une marque de considération pour l'auteur mais qu'il utilise aussi comme un moyen de revendiquer une identité professionnelle de bibliothécaire-critique par distanciation avec les critiques de presse. Cette posture est à relier à la position plus affirmée qu'il occupe dans le réseau des bibliothèques parisiennes: son rôle de secrétaire du comité d'analyse des romans, doublé de sa participation à l'opération "Premiers romans », fait de lui une personne incontournable en matière de littérature, que tout le monde connaît et reconnaît dans le réseau. II assume donc mieux le geste critique dans ses diverses modalités, adaptées aux objets singuliers. Par souci d'objectivité professionnelle et d'équité, il considère que chaque livre publié doit être au moins regardé, mais seuls lui paraissent exiger une lecture intégrale ceux qui laissent apercevoir la singularité d'un style et d'une vision :

\footnotetext{
«Beaucoup de livres échappent à la presse. Les journalistes, c'est pareil, ils sont comme nous, ils ont des piles tellement grandes sur leur table qu'ils ne font pas tout, ils choisissent. Ils en font une dizaine sur cent, alors que nous au moins, on peut garantir aux auteurs qu'ils ont été regardés, même si c'est en deux minutes et qu'on les a descendus ! [II rit] Au moins, ils ont été regardés ! [...] Quand j'ai détecté qu'il y avait un auteur, comme on dit, à ce moment-là je le reprends et je le lis en entier, voilà. » (entretien du 29 avril 2008)
}

Selon cette même exigence de professionnalisme, mais aussi pour ne pas risquer de porter ombrage à la figure naissante de l'auteur, la critique doit être la plus objective possible, visant à 
décrire plus qu'à prendre parti. La conscience d'une responsabilité du critique à l'égard de l'auteur apparaît de manière récurrente chez les bibliothécaires, en même temps que la nécessité d'une sélection permettant de faire émerger le meilleur. La bibliothécaire moins assurée qui participe à l'opération «Premiers romans » l'assume plus ou moins bien :

"Quand je fais l'analyse et que je descends en flèche un livre, parce qu'il y a des choses qui ne sont vraiment pas bonnes, je me dis : c'est fini, quoi, c'est fini parce que ça ne m'a pas plu, ça n'a plu à personne, il va y avoir très peu de tirages et il ne va pas y avoir de ventes [...] Je ne sais pas si on a bien fait de faire la critique [des livres dans le fascicule qui présente les premiers romans sélectionnés], parce que ça influence et comme la critique est très personnelle et qu'il n'y a pas de critique sans... il n'y a pas de vraie critique valable, si on peut dire : ça dépend... je ne sais pas si on a bien fait, parce que ça peut jouer en défaveur de l'auteur. » (entretien du 8 avril 2008)

Le secrétaire du comité d'analyse des romans mesure également le rôle de filtre qu'il est susceptible de jouer, mais aussi, à rebours, la visibilité qu'il peut donner à un auteur en argumentant pour qu'il figure parmi les propositions d'acquisitions. II relie cette sensibilité à sa propre position d'écrivain peu reconnu :

" J'ai aussi un regard un peu différent parce que comme je suis moimême écrivain - j'ai publié six livres, bon - donc je me dis : quelle responsabilité ! Parce qu'il m'est arrivé à certaines séances d'avoir mon livre dans les Livres Hebdo! [Il rit] Alors si vous voulez, je me sens d'autant plus responsable. Je me dis : ce pauvre écrivain qui a peut-être passé un an à écrire son livre, qui a souffert, qui a cherché un éditeur, qui l'a trouvé, bon, si je dis dis un mot de travers, il ne trouvera jamais de lecteurs. Enfin bon, il en trouvera en librairie mais c'est que le réseau des bibliothèques parisien, c'est quand même une vitrine intéressante pour un auteur. » (entretien du 29 avril 2008)

\section{Une participation au contrôle \\ du droit d'entrée dans le champ littéraire}

La lecture critique des bibliothécaires est ce qui détermine de fait ce qui est digne d'avoir sa place sur les rayons des bibliothèques, donc d'être visible et accessible pour ceux qui les fréquentent. Les bibliothécaires prétendent ainsi exercer un contrôle du droit d'entrée dans le monde des auteurs. Ce faisant, ils défendent une position dans le champ littéraire qui leur est rarement reconnue par les éditeurs (lesquels leur envoient, par exemple, peu d'ouvrages en service de presse). Le contrôle du droit d'entrée, c'est-à-dire le droit de décider qui est digne du nom d'auteur ${ }^{16}$, constitue en effet un enjeu majeur au sein du champ littéraire, à la fois pour les instances revendiquant la possibilité d'y participer et pour les producteurs qui en bénéficient ou qui aspirent à en bénéficier. C'est que le terme d'auteur ne renvoie pas seulement à une réalité éditoriale factuelle, correspondant à une position en amont de la chaîne du livre. II désigne aussi un statut symbolique ${ }^{17}$ octroyé par les instances autorisées du champ littéraire et constituant à ce titre un enjeu de lutte. Michel Foucault définit ainsi la « fonction-auteur » comme "le résultat d'une opération complexe qui construit un certain être de raison qu'on appelle l'auteur » (Foucault, 1969).

La publication assure la diffusion (GRIHL, 2002) et la responsabilité (Sapiro, 2011) mais, même à compte d'éditeur ${ }^{18}$, elle n'est qu'un premier « droit d'entrée » (Mauger, 2006) contrôlé par l'éditeur (Simonin \& Fouché, 1999). Elle ne suffit pas à constituer une identité d'auteur (Heinich, 1995), c'est-à-dire à fonder le sentiment d'être écrivain (identité pour soi), à autoriser le scripteur à se dire écrivain (présentation de soi) et surtout à assurer la reconnaissance de ce statut (identité perçue par autrui). Nathalie Heinich suggère qu'il existe un continuum du moins écrivain au plus écrivain, qui irait de l'auteur d'une seule publication peu reconnue au Grand Auteur panthéonisé. Alain Viala propose de distinguer quatre phases dans l'accès au statut d'auteur classique, qui peuvent permettre d'éclairer la seconde partie de ce continuum :

"L'accès au statut de classique constitue la forme suprême de consécration littéraire. Le procès qui y aboutit peut être décrit en quatre phases : légitimation, émergence, consécration, perpétuation.
16. Le romancier François Bégaudeau évoque avec humour cette distinction qui rappelle celle que Barthes établissait entre écrivant et écrivain : " Si comme je le souhaite ton livre est édité, si même d'autres lui succèdent, il te restera à accomplir le plus difficile : gagner le titre d'écrivain. Car, si étrange que cela te paraisse, écrivain désigne moins une activité, celle d'écrire, qu'un galon, une légion d'honneur remise virtuellement par un chœur abstrait dont le chant s'élève sur un postulat non négociable : parmi les publiés, la plupart ne sont pas écrivains. » (Bégaudeau, 2011, p. 4)

17. Cette acception se retrouve dans l'espace cinématographique à travers la catégorie de "film d'auteur».
18. La publication dite "à compte d'auteur » permet à quiconque de publier ses écrits moyennant finances. La publication par un éditeur constitue, elle, un premier filtre, qui assure un premier contrôle du droit d'entrée et autorise l'affiliation à des organismes de protection sociale. L'AGESSA définit ainsi l'auteur « comme la personne dont les œuvres imprimées sont diffusées par la voie du livre par des entreprises d'édition ayant leur siège en France (ou à l'étranger) et qui perçoit à ce titre une rémunération qualifiée de droits d'auteur » (http://www.agessa.org/telechargement/ficTelecharge_1/auteurs/ Ecrivains.pdf). 
La légitimation suppose à la fois une audience suffisante, un certain succès donc, et la reconnaissance par les pairs, par les instances autorisées du champ. L'émergence correspond au moment où l'écrivain se détache parmi un ensemble d'auteurs légitimés, se distingue, accède à une légitimation supérieure. La consécration advient quand il y a accès aux marques les plus hautes de distinction. La phase de perpétuation est, pour qu'on puisse parler de classique, des plus décisives évidemment: elle suppose l'entrée dans des espaces qui assurent une diffusion de notoriété à long terme. » (Viala, 1993, p. 25)

On propose de désigner par le néologisme d'auctorialisation le processus de constitution d'un capital symbolique et d'une identité d'auteur reconnue. Les différents acteurs du champ littéraire, professionnels et institutions, participent à divers titres à ce processus (Becker, 1988 ; Luneau \& Vincent, 2010). Partant, ils concourent à faire exister des valeurs et, simultanément, à produire la croyance dans ces valeurs (Bourdieu, 1977). Quand l'éditeur intervient en amont dans ce processus d'auctorialisation, les traducteurs (Casanova, 2002) puis les jurys de prix littéraires (Ducas, 2010) dans ses phases intermédiaires et l'institution scolaire dans la phase ultime, les bibliothécaires semblent susceptibles d'intervenir en différents moments du processus (du repérage des primo-romanciers à la perpétuation des classiques en passant par l'inscription dans la durée des auteurs plus récemment consacrés).

\section{Action culturelle}

Les bibliothécaires n'ont plus seulement affaire aux auteurs comme noms auxquels est associé un degré variable de capital symbolique, mais aussi aux écrivains comme êtres sociaux à part entière. Dans une logique de renforcement des intermédiaires du travail artistique (Lizé, Naudier \& Roueff, 2011) et de développement de "l'action culturelle », ils sont encouragés à organiser différentes formes d'animation, au nombre desquelles se trouvent les invitations d'auteurs, et qui participent à reconfigurer leur identité professionnelle.
Une nouvelle posture plus ou moins assumée d'animateur culturel

L'action culturelle en bibliothèque correspond à la conception aujourd'hui dominante des politiques culturelles, qui privilégie la culture « vivante » et l'organisation d'événements. Elle permet aussi de travailler l'image des bibliothèques en les faisant apparaître comme des lieux animés organisateurs d'événements et pas seulement des lieux banalisés du paysage culturel utilisés de manière routinière. Les municipalités mettent ainsi en avant les animations organisées, beaucoup plus qu'elles ne mentionnent le travail quotidien réalisé pour l'actualisation des collections et l'accompagnement des usagers. Ces animations ont ainsi une valeur symbolique importante, même s'il faut admettre qu'elles touchent un nombre réduit de personnes (selon les témoignages de responsables d'établissements, une invitation d'auteur dans une bibliothèque parisienne attire une quinzaine de personnes) et qu'elles ne touchent pour ainsi dire jamais un public qui ne fréquentait pas le réseau des bibliothèques préalablement (la publicité de ces manifestations étant restreinte à une diffusion dans le cadre des bibliothèques et à travers un fascicule distribué également dans les établissements, elles ne constituent pas vraiment un moyen d'ouvrir la bibliothèque à des personnes qui lui seraient étrangères).

Les animations concernant la littérature prennent notamment la forme de lectures, de projections, de conférences et de rencontres avec des auteurs (lecture de l'auteur, conférence, débat). Certaines s'inscrivent dans l'actualité d'événements littéraires plus larges comme "Le Temps de lire », les « Belles Étrangères », le "Printemps des Poètes » ou le Salon du Livre de Paris. D'autres sont organisées autour de publications ou d'acquisitions récentes. Elles sont en tout cas I'occasion de mettre en valeur une offre assez pointue et de faire apparaître la bibliothèque comme un lieu en prise sur la création littéraire, participant à l'émergence des nouveaux talents en même temps qu'à la perpétuation d'auteurs reconnus. 


\title{
ANIMATIONS LITTÉRAIRES ORGANISÉES EN BIBLIOTHÈQUES EN JANVIER ET FÉVRIER 2012
}

Source : En vue : le Magazine des Bibliothèques de la ville de Paris, N 52. Classement fait par l'auteur.

\section{AUTEURS CLASSIQUES}

\author{
1. Analyses \\ Projection d'un documentaire sur Georges Perec. \\ Conférence sur Stendhal. \\ Conférence sur le poète René-Guy Cadou. \\ 2. Lectures et mises en scène \\ Mise en scène (théâtre de papier) du conte Macao et Cosmage (1919). \\ Lecture de poèmes d'Arthur Rimbaud, Une saison en enfer. \\ Lecture de la nouvelle Le Bal d'Irène Némirovsky (1930).
}

\section{LITTÉRATURE CONTEMPORAINE}

\author{
1. Lectures \\ Lecture d'extraits de littérature cubaine (Wendy Guerra, Zoé Valdès, Reinaldo Arenas...). \\ Lecture d'extraits du conte Le Centaure dans le jardin du Brésilien Moacyr Scliar. \\ Lecture d'extraits du roman contemporain 1 Q84 de Haruki Murakami. \\ 2. Rencontres \\ Rencontre avec l'auteur de bande dessinée militante Pierre Maurel. \\ Rencontre avec trois auteurs inspirés par la musique : Lydie Salvayre (Hymne, sur Jimi Hendrix), William Memlouk (Mingus Mood, sur Charles \\ Mingus), Renaud Czarnes (premier roman, héros musicien de jazz). \\ Rencontre avec quatre auteurs ou artistes qui mettent Paris en fiction : Gérard Mordillat (récit autobiographique), Jacques Jouet (poésie), Marc \\ Villard (romans noirs), Cyrille Derouineau (photographie). \\ Veillée de contes.
}

La posture d'animateur culturel ne va cependant pas de soi pour des bibliothécaires qui y sont peu, voire pas du tout formés et ne bénéficient que de moyens financiers limités pour mener à bien ces actions. Une bibliothécaire témoigne ainsi des divisions que suscite ce nouveau rôle au sein de son équipe entre «ceux qui sont pour l'animation » et «ceux qui sont contre » : "Moi, je suis pour l'animation, parce que je pense qu'il n'y a pas que le livre qui compte et que sinon, bon, à ce moment-là, on met des automates de prêt et puis on ne s'occupe plus de rien, on ne va pas conseiller les lecteurs, on ne va pas les encourager à chercher les livres sur les rayons ou à essayer de trouver des choses nouvelles » (entretien du 8 avril 2008). Cette nouvelle posture d'animateur suscite d'autant plus de réticences qu'on est ici dans une grande bibliothèque d'un quartier universitaire qui accueille essentiellement un public d'étudiants et d'intellectuels. Elle s'impose davantage dans les établissements qui cherchent à modifier l'image de la bibliothèque auprès du grand public. Mais souvent des modérateurs extérieurs sont invités à dialoguer avec les auteurs, tandis que les bibliothécaires sont les organisateurs qui restent dans l'ombre: "Quand on invite nos auteurs, on essaie de regrouper deux auteurs qui sont à peu près semblables avec un modérateur - parce que nous, en principe, dans les bibliothèques on ne sert pas de modérateur ». L'organisation de rencontres avec les auteurs permet ainsi de questionner le statut d'auteur tel qu'il est construit par ces manifestations, mais aussi de réinterroger à nouveaux frais l'identité professionnelle des bibliothécaires telle qu'elle se reconstruit dans cette nouvelle relation à l'auteur. 


\section{L'homme pour la promotion de l'œuvre}

Proust reprochait à Sainte-Beuve de « ne pas avoir vu l'abîme qui sépare l'écrivain de l'homme du monde, [de] n'avoir pas compris que l'écrivain ne se montre que dans ses livres ${ }^{19}$. L'explication de l'œuvre par l'homme semble aujourd'hui une approche dépassée et pourtant la tendance à rapporter des productions textuelles à la personne sociale de leur producteur demeure une modalité courante d'approche des textes tandis que l'organisation de rencontres avec des auteurs participe à la promotion de leurs dernières publications. Les invitations d'auteurs en bibliothèque s'inscrivent dans les "activités paralittéraires » qui constituent un volet de la « double vie des écrivains » et un des signes de reconnaissance du statut d'écrivain (Lahire, 2006, pp. 212-230). Elles peuvent aussi se lire comme une manifestation de «l'économie du star-system »(Benhamou, 2002) qui se répand dans le champ éditorial et dans laquelle un livre ne se suffit plus à lui-même. L'auteur doit mettre de sa personne pour se faire connaître et repérer, accorder des entretiens à des journalistes, se livrer à une surenchère de séances de signatures en librairies (Thorel, 2008) et dans des salons et festivals : il sert alors de faire-valoir à son livre. L'exigence de rentabilité imposée par les éditeurs oblige l'auteur à se plier à cette logique d'exploitation commerciale de sa personne, sous peine de ne pas vendre suffisamment et de voir ses prochains textes refusés.

Mais en utilisant une technique de promotion, les bibliothécaires en inversent la logique: les auteurs invités sont rarement des stars (qui sans doute ne se déplaceraient pas), mais plutôt des écrivains en voie de légitimation. Ils entendent ainsi donner une visibilité à ce qui en a peu, dans un contexte de surproduction éditoriale et de domination de l'espace médiatique par un petit nombre d'auteurs phares tendant à attirer sur eux l'attention générale. À la différence de l'optique des dédicaces dans les grandes surfaces culturelles ou les salons, l'objectif n'est pas d'assurer des ventes importantes en jouant d'un effet de vedette, mais très modestement d'inviter un petit cercle d'usagers à découvrir un auteur qui mérite d'être connu et, partant, de constituer la valeur même de cet auteur, tout en travaillant l'image de la bibliothèque.
II s'agit en effet aussi de montrer que la bibliothèque n'est pas un cimetière d'auteurs disparus, de donner à l'auteur une réalité plus tangible et, en déplaçant l'accent de l'œuvre existante à son créateur, de mettre en avant le travail d'écriture et le geste de création : "On trouve que ce n'est pas trop mal que les gens puissent voir en chair et en os des auteurs. ${ }^{20}$ " L'invitation d'un petit nombre d'auteurs constitue ainsi le couronnement de l'opération «Premiers romans ». La plupart des auteurs invités se prêtent au jeu de la rencontre, conscients que "ça peut aider d'être en bibliothèque », selon les termes du secrétaire du comité romans. Quelques uns s'y refusent, considérant sans doute que cela "casse tout ", selon l'expression d'une bibliothécaire participant à l'opération : « II a pondu ça, j'allais dire [Elle rit], il l'a donné, il ne voulait pas faire autre chose, il ne voulait pas l'expliquer. II n'y avait rien à expliquer de toute façon : quand on lit, on lit et puis après on interprète comme on veut. » (entretien du 8 avril 2008)

\section{L'homme pour éclairer l'œuvre?}

"L'explication de l'œuvre est toujours cherchée du côté de celui qui l'a produite, comme si, à travers l'allégorie plus ou moins transparente de la fiction, c'était toujours finalement la voix de l'auteur, qui livrait sa "confidence" », regrettait Barthes (Barthes, 1968, p. 62), pour qui les significations du texte devaient être recherchées par-delà les intentions de l'auteur. L'organisation de rencontres-débats entre un auteur et ses lecteurs rompt avec le schéma de communication in absentia qui caractérise l'écriture et la lecture littéraires. Elle permet d'accéder à la parole de l'auteur et, partant, à son intention, non pas telle qu'elle est réalisée dans le livre, échappant plus ou moins à la conscience de l'écrivain lui-même, mais telle qu'elle apparaît à la conscience de l'auteur lui-même (Compagnon, 1999). Elle place l'auteur dans la position de garant du sens de son propre texte, légitime à participer au travail d'interprétation de son œuvre, à la manière des plasticiens d'art contemporain. La bibliothécaire déjà citée oscille entre un tel point de vue et celui, apparemment antithétique, de la liberté du lecteur qui rend chaque interprétation singulière aussi valable qu'une autre :
19. Dans un texte intitulé Disparaître, l'écrivain et critique Bertrand Leclair reprend ce thème du dédoublement entre l'écrivain qui écrit et celui qui parle de son texte dans une rencontre littéraire: "Mais enfin, ici, face à vous, au mieux je suis l'agent double de celui-là qui écrit, d'un côté l'autre du rideau des mots » (Leclair, 2004).
20. Bibliothécaire déjà citée impliquée dans l'opération «Premiers romans ». 
"L'intérêt [de rencontrer un auteur] c'est que... On le lit comme on veut, mais on voudrait être sur la même longueur d'onde que l'auteur. On voudrait savoir si on n'a pas raté quelque chose ou si on a bien compris où il voulait aller. Et on a des informations sur l'auteur, sur sa vie, sur sa façon d'écrire, sur sa façon de... Pourquoi il a fait ce livre? Comment il a été publié ? Qu'est-ce qu'il a comme objectif, comme projet? Ça c'est aussi quelque chose qui serait très intéressant sur tous les livres de la bibliothèque. Mais comme on ne connaît rien sur cet auteur, pratiquement rien, eh bien... et que souvent il est heureux de pouvoir partager avec le lecteur, de partager quelque chose, entre autres il partage sa vie, il partage ce qu'il a voulu donner, [...] pour moi, c'est intéressant. [...] Et nous on voit que vraiment, quand l'auteur parle de son livre, on n'a même pas compris un dixième de ce qu'il voulait dire, et encore, hein. » (entretien du 8 avril 2008)

Le lecteur est néanmoins considéré comme capable, en s'appropriant le texte dans la solitude du tête-à-tête avec le livre, de dégager par lui-même des significations qui peuvent avoir pour partie échappé à l'intention consciente de l'auteur.

\begin{abstract}
" Ça, c'est des choses, on se demande pourquoi ils ont fait ça et à chaque fois on comprend bien. Parce que souvent ils disent: "J'ai voulu faire ça pour telle et telle raison." Mais souvent ils disent: "Je n'ai pas du tout voulu vous faire comprendre comme ça, ou vous faire connaître ça. Comment vous le savez ?" Ils disent quelquefois : "Mais comment vous l'avez su?" Ben on l'a lu dans son livre... [...] Celui qui a écrit toutes ces pages, il dit: "Mais comment vous le savez?" Franchement on se dit: "Ben voilà, c'est parce qu'on a lu entre les lignes ou qu'on a deviné". [...] Ça fait un peu salon littéraire de voir quelqu'un qui vient expliquer son œuvre. [...] Celui qui a envie de lire un livre et de relire un livre, il n'a pas besoin d'avoir un spectacle ou d'avoir du contact, il est seul avec son ouvrage et il le lit quand il veut, où il veut, à haute voix ou dans sa tête. Et ça ne change rien à son impression qu'il a eue de la lecture, même si on lui explique que la personne qui a écrit, c'est quelqu'un de formidable et puis qui a toutes les qualités de la terre [Elle rit], ça ne changera pas son impression de lecture, même si on lui dit : “Eh bien, vous voyez, vous n'avez pas aimé ce livre-là, mais l'auteur...", ça ne changera pas non plus. » (ibid.)
\end{abstract}

II ne s'agit pas tant d'imposer au lecteur une interprétation « vraie » qui serait conforme à l'intention consciente de l'auteur que de " faire lire les gens et [de] leur faire trouver leur propre jugement », de « donner envie de lire, parce qu'il y a des gens qui n'ont pas lu les livres, donner envie de les acheter, et puis discuter de la littérature, discuter de l'écriture et susciter l'envie. »Dans une inversion de perspective, c'est plutôt à l'auteur que la rencontre est censée pouvoir profiter (surtout si c'est un primo-romancier) en lui faisant voir son propre texte sous un jour différent :

"Je me dis que pour un auteur, c'est important qu'il voie la façon dont ça a été appréhendé, ce qu'il a écrit. Ça, c'est très très important. Parce que, quand on parle avec un auteur, il dit: "Ah, je ne me rappelle pas trop avoir écrit ça..." Parce que quand on écrit, on ne voit pas de la même façon. "Ah, vous pensez... ça, ça vous a touché ? Ah, je ne m'en étais pas rendu compte." [...] Les auteurs sont toujours surpris que les gens aient vu des tas de choses dans leur écriture alors qu'ils n'ont pas forcément voulu dire tout ça. Ils n'ont pas vraiment voulu dire tout ça. "Ah, ils me disent, vous avez compris ça comme ça, ah oui... Ah, ça donne cette impression ?" Comme quelqu'un qui aurait fait une œuvre d'art sans s'en rendre compte. Voilà. Ils ne se rendent pas compte de la portée que ça peut avoir : ils sont encore trop naïfs ou trop jeunes dans l'écriture. » (ibid.)

Une telle perception recoupe les résultats de l'enquête de Bernard Lahire, dans laquelle 61,4 \% des écrivains interrogés jugent « intéressantes » les interventions en bibliothèque (Lahire, 2006, p. 601).

C'est donc bien la lecture et le travail interprétatif qu'elle suppose qui constituent l'œuvre littéraire comme telle et, partant, font du scripteur un auteur. La rencontre entre lecteurs et auteurs participe alors à constituer l'auteur, mais aussi le lecteur, dans une division du travail où les rôles sont nettement distribués.

\section{Une division du travail entre auteurs et lecteurs}

La rencontre entre auteurs et lecteurs humanise les auteurs et favorise l'empathie des lecteurs à l'égard d'individus qui semblent descendre de leur piédestal d'écrivains pour apparaître comme des semblables, des "gens dans le monde", qui «font ça en plus de leur travail ». Mais la rencontre est aussi placée sous le signe de la distance : l'écrivain n'est pas n'importe qui et n'importe qui n'est pas écrivain, comme le souligne la bibliothécaire déjà citée en mobilisant successivement l'idéologie du don puis l'argument de la difficulté : «Tout le monde ne peut pas écrire, hein. C'est quand même un don. Enfin, un don... C'est beaucoup de travail, je disais, mais c'est quand même aussi un don. Donc ce n'est pas venu comme ça sans difficulté, hein. » II ne s'agit donc pas de susciter chez le lecteur l'envie d'écrire ou de l'inviter à se lancer lui-même dans l'écriture. La position occupée par l'auteur dans la rencontre cantonne le lecteur dans sa position de récepteur et le persuade de sa propre incapacité à occuper l'autre position, au-delà des désirs réprimés qu'il peut découvrir en lui. Ainsi la bibliothécaire qui a admis que « tout le monde ne peut pas écrire " s'avoue tentée par l'écriture mais trouve des justifications pour expliquer qu'elle ne passe pas à la pratique :

« Je m'intéresse à la pratique d'écriture comme la plupart des gens qui s'intéressent au premier roman, hein. Ils auraient envie d'écrire, 
ils ont envie un peu mais... Moi, je ne dis pas que je n'écrirais pas, j'ai déjà écrit des petits textes, mais je ne sais pas. [...] D'un certain côté, ça m'intéresserait de faire ça, d'écrire quelque chose, mais d'un autre côté je ne vois pas vraiment à quoi ça me servirait, à part me soulager de quelque chose ou laisser un témoignage précis sur un moment de ma vie. » (entretien du 8 avril 2008)

L'approche scolaire de la littérature (Jey, 1998 ; Schmitt, 1990 ; Baudelot, Cartier \& Détrez, 1999), y compris dans ses formes modernes (Burgos \& Privat, 1993), n'est pas pour rien dans l'incorporation du principe de division du travail entre les auctores, producteurs de textes, et les lectores, qui les lisent et éventuellement les commentent (Bourdieu \& Chartier, 2003, p. 278) mais qui sont persuadés de l'inaccessibilité de la position d'auctor pour eux qui n'ont pas reçu le « don » et la « vocation » de l'écriture. Elle semble en tout cas avoir été décisive dans le cas évoqué ci-dessus :

«Quand j'étais en classe en troisième, j'avais un professeur de français qui était extraordinaire - j'étais au lycée Montaigne - et il nous a dit une chose - pourtant ça fait longtemps, hein, c'était avant 68 - qui m'a complètement marquée pour toute ma vie. II a dit : c'est très facile d'écrire sa vie, le plus difficile c'est d'intéresser les autres. Eh bien là, quand je lis le premier roman, je me dis ce n'est pas si facile d'écrire sa vie, ça n'intéresse pas toujours les autres. Je pense que je n'ai pas en moi quelque chose qui fait que ce soit aussi important à partager avec d'autres. Mais j'aimerais l'écriture, la façon de rédiger, de travailler les mots, de faire des sortes de phrases qui sont joliment posées, qui sont présentées, qui sont agréables à entendre. Mais ça ne serait que du toc ! [Elle rit] Parce que la vraie chose dedans, je n'ai pas encore trouvé. Si je le trouve un jour, quelque chose qui soit suffisamment fort pour que ça m'oblige à faire tout l'effort nécessaire, intellectuel et puis l'effort physique, l'énergie à donner, pour pouvoir croire que j'ai... Ça serait prétentieux de ma part actuellement de dire : je dois écrire. À moins qu'il y ait quelque chose qui vienne sur ma tête brutalement! [Elle rit] Je ne sens pas le livre en attente qui va sortir coûte que coûte, qu'il faudra avoir comme un accouchement. » (ibid.)

Séparé de l'auteur par un fossé infranchissable, le lecteur, qu'il soit usager ou bibliothécaire, est en posture de réception et d'admiration respectueuse et curieuse, jamais de production littéraire. La lecture est conçue comme sa propre fin et non comme une source d'inspiration pour l'écriture. Si la bibliothèque accueille des rencontres avec des auteurs, c'est presque toujours pour évoquer des œuvres déjà publiées: il n'est pas question d'organiser des ateliers d'écriture qui encourageraient l'usager à se faire auteur (Chateigner, 2008) et viendraient nourrir l'illusion d'une possibilité pour les profanes de franchir les frontières du champ littéraire (Poliak, 2006). Même le secrétaire du comité romans, malgré sa position plus assurée et ses six livres publiés, ose à peine prétendre au titre d'auteur :

\begin{abstract}
" Je vais souvent dans des soirées littéraires comme auditeur pour écouter les auteurs et notamment dans des cafés littéraires [...]. Je n'ose même pas dire que je suis un auteur, je suis quelqu'un qui a eu la chance de publier à compte d'éditeur, ce qui est déjà bien. [... En lisant], je ne me pose jamais la question, que je trouverais complètement idiote d'ailleurs, de : "Ah, j'aurais pu l'écrire!" ou "Je n'aurais jamais écrit ça” parce que... pff... déjà que c'est difficile de se mettre à la place d'un lecteur, je ne vais pas en plus me mettre à la place d'un lecteur-écrivain. » (entretien du 29 avril 2008)
\end{abstract}

Ainsi, l'échange entre auteurs et lecteurs, comme la lecture, maintient strictement la division du travail entre les uns et les autres et, partant, participe à constituer la posture de l'auteur comme singulière et distinctive et celle du bibliothécaire comme médiateur dominé.

\section{Conclusion}

La figure de l'auteur n'est donc pas un simple donné pour les bibliothécaires, mais le résultat d'une construction à laquelle ils participent de différentes manières, par l'importance qu'ils attachent au capital symbolique attaché à un nom propre, mais aussi en situant chaque livre dans la perspective d'une œuvre et d'une trajectoire auctoriale, et par la manière dont ils conçoivent et organisent les invitations d'auteurs en bibliothèque. Comme institution de la vie littéraire, la bibliothèque de lecture publique prend ainsi part au processus d'auctorialisation en ses différentes phases et dimensions, du repérage des nouveaux talents à la perpétuation de la consécration, en passant par la confirmation et le prolongement d'une légitimation conférée par d'autres instances : elle constitue à ce titre un sas de l'histoire littéraire. La manière spécifique dont se déroule cette auctorialisation joue sur la triple temporalité de la bibliothèque (prise entre temps du canon et temps de l'actualité éditoriale et insistant plus particulièrement sur la temporalité médiane) et sur une triple identité professionnelle (de lettré, de critique et d'animateur). Ainsi, non contents de mettre en œuvre les politiques publiques de la lecture, les bibliothécaires participent aussi, ce faisant, aux luttes de positions et de définition de valeurs qui animent le champ littéraire, même s'ils y occupent une position relativement invisible (Naudier, 2010) et en tout cas dominée. 


\section{Bibliographie}

Abensour C. \& Legendre B. (2012), Entrée en littérature : premiers romans et primo-romanciers dans les limbes, Paris, Éd. Arkhê.

Abbott A. (1988), The System of Professions: An Essay on the Division of Expert Labor, Chicago, The University of Chicago Press.

Bahloul J. (1988), Lectures précaires : étude sociologique sur les faibles lecteurs, Paris, BPI.

Barthes R. (1984 [1968]), "La mort de l'auteur", in Le Bruissement de la langue : essais critiques IV, Paris, Éd. du Seuil.

Baudelot C., Cartier M. \& Détrez C. (1999), Et pourtant ils lisent, Paris, Éd. du Seuil.

Bayard P. (2007), Comment parler des livres que l'on n'a pas lus, Paris, Éd. de Minuit.

Becker H. (1988), Les Mondes de l'art / trad. de l'anglais par Jeanne Bouniort, Paris, Flammarion (trad. de Art Worlds, The Regents of the University of California, 1982).

Bégaudeau F. (2011), Tu seras écrivain mon fils, Paris, Éd. Bréal. Benhamou F. (2002), L'économie du star-system, Paris, Odile Jacob.

Bertrand A.-M. (2004), « Approche archéologique et généalogique du métier » in Calenge B. (dir.), Bibliothécaire, quel métier?, Paris, Éd. du Cercle de la Librairie.

Bourdieu P. (1977), «La production de la croyance : contribution à une économie de la production des biens symboliques ", Actes de la recherche en sciences sociales, n 13, pp. 3-43.

Bourdieu P. (1979), La Distinction : critique sociale du jugement, Paris, Éd. de Minuit.

Bourdieu P. (1991), "Le champ littéraire", Actes de la recherche en sciences sociales, n० 89, pp. 3-46.

Bourdieu P. \& Chartier R. (2003 [1985]), « La lecture : une pratique culturelle » in Chartier R. (dir.), Pratiques de la lecture, Payot \& Rivages "Petite bibliothèque Payot ».

Bourdieu P. \& Delsaut Y. (1975), "Le couturier et sa griffe: contribution à une théorie de la magie ", Actes de la recherche en sciences sociales, n 1 , pp. 7-36.

Burgos M. \& Privat J.-M. (1993) « Le Goncourt des lycéens : vers une sociabilité littéraire? ", in Poulain M. (dir.), Lire en France aujourd'hui, Paris, Éd. du Cercle de la Librairie, pp. 163-181.

Casanova P. (1999), La République mondiale des lettres, Paris, Éd. du Seuil.

Casanova P. (2002), " Consécration et accumulation de capital littéraire : la traduction comme échange inégal », Actes de la recherche en sciences sociales, n ${ }^{\circ}$ 144, pp. 7-20.

Chartier A.-M. \& Hébrard J. (2000), Discours sur la lecture (1880-2000), Paris, BPI-Fayard.

Chateigner F. (2008), Une Société littéraire : sociologie d'un atelier d'écriture, Paris, Éd. du Croquant.

Compagnon A. (1999), Le Démon de la théorie, Paris, Éd. du Seuil.

Conseil Supérieur des Bibliothèques (1991), Charte des bibliothèques, en ligne http://www.enssib.fr/bibliotheque-numerique/ document-1974.

Dubar C. (2000), La Crise des identités : l'interprétation d'une mutation, Paris, PUF.
Dubois V. (1999), La Politique culturelle : genèse d'une catégorie d'intervention publique, Paris, Belin.

Ducas S. (2010), « Prix littéraires en France : consécration ou désacralisation de l'auteur? », ConTextes, n 7.

Foucault M. (2001 [1969]), « Qu'est-ce qu'un auteur? », Dits et écrits I, 1954-1975, Paris, Gallimard « Quarto », pp. 817-840.

Gleize J. \& Roussin Ph. (dir.) (2009), La Bibliothèque de la Pléiade : travail éditorial et valeur littéraire, Paris, Éditions des archives contemporaines.

GRIHL (2002), De la publication, Paris, Fayard.

Heinich N. (1995), "Façons d'être écrivain : l'identité professionnelle en régime de singularité ", Revue française de sociologie, vol. 36, n³, pp. 499-524.

Heinich N. (2000), Etre écrivain : création et identité, Paris, La Découverte "L'Armillaire».

Jauss H. R. (1978), Pour une esthétique de la réception / trad. par Cl. Maillard, Paris, Gallimard.

Jey M. (1998), La Littérature au lycée : invention d'une discipline, 1880-1925, Metz, Université de Metz.

Lahire B. (2006), La condition littéraire : la double vie des écrivains, Paris, La Découverte.

Leclair B. (2004), Disparaître, Tours, Farrago \& Léo Scheer.

Lizé W., Naudier D. \& Roueff 0. (2011), Intermédiaires du travail artistique : à la frontière de l'art et du commerce, Paris, Département des études, de la prospective et des statistiques, DL.

Luneau M.-P. \& Vincent J. (dir.) (2010), La Fabrication de l'auteur, Québec, Éditions Nota Bene.

Mauger G. (dir.) (2006), Droits d'entrée: modalités et conditions d'accès aux univers artistiques, Paris, Éd. de la Maison des sciences de l'homme.

Mauger G. \& Poliak C. (1998), « Les usages sociaux de la lecture ", Actes de la recherche en sciences sociales, n 123 , pp. 3-24.

Naudier D. (2010), "Les attachées de presse : les maillons invisibles de l'édition ", Document de travail du Mage, nº13, pp. 35-46, en ligne.

Olivero I. (1999), L'invention de la collection : de la diffusion de la littérature et des savoirs à la formation du citoyen au XIXe siècle, Paris, IMEC-MSH.

Parmentier P. (1986), "Les genres et leurs lecteurs », Revue française de sociologie, vol. 28, n 3, pp. 397-430.

Poliak C. (2006), Aux frontières du champ littéraire : sociologie des écrivains amateurs, Paris, Éd. Économica.

Ponton R. (2004), "Auteur » ; "Écrivain », in Aron P., SaintJacques D. \& Viala A. (dir.), Le dictionnaire du littéraire, Paris, Presses Universitaires de France, 2002, rééd. "Quadrige », pp. $22-34$; 172-174.

Rabot C. (2011a), "Les "Coups de cœur" d'une bibliothèque de lecture publique : valeurs et enjeux professionnels d'une sélection littéraire ", Culture \& Musées, n 17, pp. 63-84.

Rabot C. (2011b), Les Choix des bibliothécaires ou la fabrication des valeurs littéraires en bibliothèque de lecture publique, thèse de doctorat, Université Paris Sorbonne Nouvelle.

Sapiro G. (2011), La Responsabilité de l'écrivain : littérature, droit et morale en France (XIXe-XXle siècle), Paris, Éd. du Seuil. 
Schmitt M. P. (1990), Fictions de la lecture: de la formation des goûts littéraires dans l'enseignement secondaire : contribution à une histoire littéraire de notre temps / thèse de doctorat, Université Paris 3 Sorbonne Nouvelle.

Seibel B. (1992), " Les enjeux d'une profession » in Poulain M. (dir.), Histoire des bibliothèques françaises 4: Les bibliothèques au XXe siècle, 1914-1990, Paris, Promodis - Éd. du Cercle de la librairie.

Simonin A. \& Fouché P. (1999), « Comment on a refusé certains de mes livres : contribution à une histoire sociale du littéraire », Actes de la recherche en sciences sociales, n 126-127, pp. 103-115.

Tarin L. (2006), " L'évolution du métier de bibliothécaire : une identité professionnelle à multiples facettes », in Bertrand A.-M.
\& Le Saux A. (dir.), Regards sur un demi-siècle : cinquantenaire du Bulletin des Bibliothèques de France / hors-série du Bulletin des Bibliothèques de France.

Thiesse A.-M. (2000 [1984]), Le Roman du quotidien : lecteurs et lectures populaires à la Belle-Époque, Paris, Le Chemin vert.

Thorel C. (2008), "Libraires et écrivains", in Leblanc F. \& Sorel P. (dir.), Histoire de la librairie française, Paris, Éd. du Cercle de la Librairie, pp. 587-593.

Viala A. (1985), Naissance de l'écrivain : sociologie de la littérature à l'âge classique, Paris, Éd. de Minuit.

Viala A. (1993), "Qu'est-ce qu'un classique?», Littératures classiques, no 19, pp. 13-31. 